\title{
Extracorporeal membrane oxygenation support improves survival of patients with severe Hantavirus cardiopulmonary syndrome
}

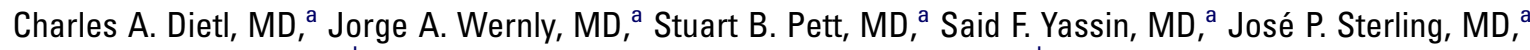 \\ Robert Dragan, BS, CCP, Karen Milligan, RN $^{\mathrm{c}}$ and Mark R. Crowley, $\mathrm{MD}^{\mathrm{d}}$
}

From the Division of Thoracic and Cardiovascular Surgery, Department of Surgery, ${ }^{2}$ Perfusion Services, ${ }^{\mathrm{b}}$ ECMO Services, ${ }^{\mathrm{c}}$ and Division of Pediatric Critical Care, Department of Pediatrics, ${ }^{\mathrm{d}}$ University of New Mexico Health Sciences Center, Albuquerque, NM.

Read at the Thirty-third Annual Meeting of The Western Thoracic Surgical Association, Santa Ana Pueblo, NM, June 27-30, 2007.

Received for publication June 20, 2007; revisions received Aug 19, 2007; accepted for publication Nov 13, 2007

Address for reprints: Charles A. Dietl, MD, Thoracic and Cardiovascular Surgery, Department of Surgery (ACC-2), University of New Mexico Health Sciences Center, MSC 10 5610, Albuquerque, NM 871310001 (E-mail: cdietl@comcast.net).

J Thorac Cardiovasc Surg 2008;135:579-84 $0022-5223 / \$ 34.00$

Copyright $\odot 2008$ by The American Association for Thoracic Surgery

doi:10.1016/j.jtcvs.2007.11.020
Objective: The purposes of this study are to evaluate the outcome of extracorporeal membrane oxygenation support in a subgroup of patients with Hantavirus cardiopulmonary syndrome who had a predicted mortality of $100 \%$ and to assess the complications associated with this treatment modality and with different cannulation techniques.

Methods: Thirty-eight patients with severe Hantavirus cardiopulmonary syndrome were supported with extracorporeal membrane oxygenation between April 1994 and June 2006. Cannulation of the femoral vessels was performed on an emergency basis by a percutaneous approach in $15(39.5 \%)$ and by an open technique in 23 (60.5\%) patients. Duration of extracorporeal membrane oxygenation averaged 132 hours (range: 5-276 hours).

Results: Complications from percutaneous cannulation occurred in 4 (26.6\%) of 15 patients: retroperitoneal hematoma in $2(13.3 \%)$ and lower extremity ischemia in 2 (13.3\%) patients, which resolved after insertion of a distal perfusion cannula. Complications from open femoral cannulation occurred in 8 (34.8\%) of 23 patients: severe bleeding in $7(30.4 \%)$ patients and lower extremity ischemia in $1(4.3 \%)$ patient who required a leg amputation. The overall survival was $60.5 \%$ (23/38 patients). Six $(40 \%)$ of the 15 patients cannulated percutaneously and $9(39.1 \%)$ of 23 patients who had open cannulation died. All survivors recovered completely and were discharged from the hospital after a mean hospital stay of 20.8 days (range: 10-39 days).

Conclusions: Almost two thirds of the patients with severe Hantavirus cardiopulmonary syndrome who were supported with extracorporeal circulation survived and recovered completely. The complications associated with both types of femoral cannulation may be attributed to the fact that all patients were in shock or in full cardiac arrest, and the procedure had to be done expeditiously. Earlier institution of extracorporeal membrane oxygenation may decrease the complication rates and improve the overall survival.

$\mathrm{H}$ antaviral infection was first recognized in the Four Corners region of the American southwest in 1993, during an outbreak of unexplained deaths in previously healthy young individuals in whom acute onset of respiratory failure and pulmonary edema developed. ${ }^{1}$ This clinical syndrome was initially called Hantavirus pulmonary syndrome. Since most deaths are caused by myocardial dysfunction and hypoperfusion, several investigators prefer the term Hantavirus cardiopulmonary syndrome (HCPS). ${ }^{2}$ The Sin Nombre virus (SNV) has been identified as the etiologic agent of HCPS and is carried by the deer mouse Peromyscus maniculatus. The SNV is transmitted primarily by inhalation of urine and feces of contaminated rodents. ${ }^{3}$ Currently, there are no antiviral drugs, vaccines, or immunotherapeutic agents available for treatment of the disease, and therapy is primarily supportive. ${ }^{4} \mathrm{De}-$ spite greater awareness and recognition of HCPS and improvement in critical care 


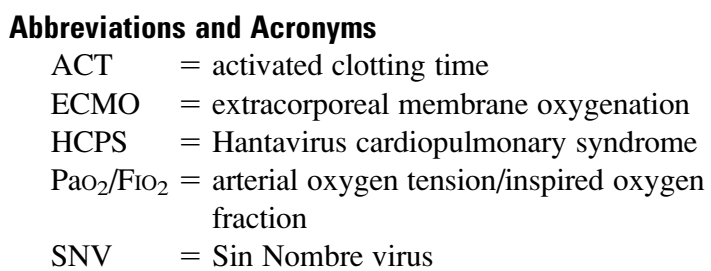

management, mortality rates from $43 \%$ to $76 \%$ have been reported. ${ }^{5,6}$ In 1998, Crowley and associates ${ }^{7}$ reported our initial experience with the use of venoarterial extracorporeal membrane oxygenation (ECMO) as a rescue therapy in 3 patients with severe cardiopulmonary failure owing to HCPS. The purpose of the present study is to evaluate the outcome of ECMO support in a subgroup of patients with HCPS who had a predicted mortality of $100 \%$ and to assess the complications associated with this treatment modality and with different cannulation techniques.

\section{Patients and Methods}

\section{Patient Selection}

We reviewed retrospectively the medical records of 38 consecutive patients with severe cardiopulmonary failure resulting from hantaviral infection treated with venoarterial ECMO at the University of New Mexico Health Sciences Center from April 1994 to June 2006. Approval by the institutional review board was obtained, and informed consent was obtained from each patient or the closest relative.

The University of New Mexico Health Sciences Center is the referral center for patients with the presumed diagnosis of Hantavirus infection. Patients were referred from the Four Corners region (New Mexico, Arizona, Colorado, and Utah) presenting with a brief history of fever, headache, and myalgias, followed by rapid deterioration, characterized by respiratory distress, and diffuse pulmonary infiltrates on chest radiograph. Most patients had a history of exposure to rodent droppings while cleaning a shed or a building 1 or 2 weeks before experiencing symptoms. Early presumptive diagnosis of Hantavirus infection was strongly suspected by the presence of thrombocytopenia, hemoconcentration, myelocytosis, and at least $10 \%$ lymphocytes with immunoblastic features in the peripheral blood smear. ${ }^{8}$ Serologic tests were positive for SNV antibodies in $100 \%$ of the patients. All patients were observed in the intensive care unit, with a radial artery line and a hemodilution catheter (Swan-Ganz; Edwards LifeSciences, Irvine, Calif) inserted through the left subclavian vein (to have the right side of the neck available for possible cannulation of the right internal jugular vein). Arterial blood gases, serum lactate levels, and cardiac index were recorded frequently. Several patients needed supportive treatment only and were not included in this study.

Most patients with HCPS went into shock precipitously, and the cardiac index was unobtainable at the time of cannulation. Selection criteria for ECMO support are summarized in Table 1. The current inclusion criteria for starting patients on ECMO are as follows: a clinical presentation consistent with HCPS, a cardiac index that
TABLE 1. Criteria for starting ECMO (In addition to clinical presentation consistent with HCPS and a cardiac index less than $2.0 \mathrm{~L} \cdot \min ^{-1} \cdot \mathrm{m}^{-2}$ )

$\begin{array}{lr}\text { Refractory shock } & 38(100 \%) \\ \text { Severe hypoxia }\left(\mathrm{PaO}_{2} / \mathrm{F}_{10} \text { ratio }<60\right) & 23(60.5 \%) \\ \text { External cardiac massage } & 8(21.0 \%)\end{array}$

ECMO, Extracorporeal membrane oxygenation; HCPS, Hantavirus cardiopulmonary syndrome; $\mathrm{PaO}_{2} / \mathrm{F}_{10}{ }_{2}$, arterial oxygen tension/inspired oxygen fraction.

rapidly drops to less than $2.0 \mathrm{~L} \cdot \mathrm{min}^{-1} \cdot \mathrm{m}^{-2}$, an arterial oxygen tension/inspired oxygen fraction $\left(\mathrm{PaO}_{2} / \mathrm{FIO}_{2}\right)$ ratio of less than 60 , or cardiopulmonary deterioration with refractory shock despite maximum inotropic support. Only 1 patient, who was 75 years old and was in multiorgan system failure and neurologically compromised before transfer, was not considered for ECMO support.

Thirty-eight patients who did not respond to maximal conventional medical therapy were cannulated and placed on ECMO on an emergency basis. They are the basis of this report. They represent approximately $75 \%$ of the patients treated for HCPS at our institution. Their ages ranged from 11 to 69 years (mean age: 41.9 years) (Figure 1). There were $21(55 \%)$ female and $17(45 \%)$ male patients. On admission to the hospital, 13 (34.2\%) patients were in hemodynamically stable condition but required increasing oxygen concentrations. The other $25(65.8 \%)$ patients were intubated before transfer to our hospital. Before cannulation, 38 (100\%) patients were receiving inotropic agents because of hemodynamic deterioration, and $8(21 \%)$ required external cardiac compressions.

Pre-ECMO mean arterial pressure ranged from 0 to $87 \mathrm{~mm} \mathrm{Hg}$ (mean: $52 \mathrm{~mm} \mathrm{Hg}$ ); $\mathrm{Po}_{2}$ ranged from 28 to $154 \mathrm{~mm} \mathrm{Hg}$ (mean $\mathrm{PO}_{2}: 65 \mathrm{~mm} \mathrm{Hg}$ ); $\mathrm{pH}$ ranged from 6.89 to 7.49 (mean $\mathrm{pH}: 7.31$ ); serum lactate level ranged from 1.1 to $20.9 \mathrm{mmol} / \mathrm{L}$ (mean lactate level: $7.3 \mathrm{mmol} / \mathrm{L}$ ); and platelet count ranged from 12,000 to 63,000 (mean platelet count: 32,000).

Patients were cannulated for ECMO when their clinical condition deteriorated, as indicated previously. Thirteen (34.2\%) patients who were not intubated before arrival were intubated at the same

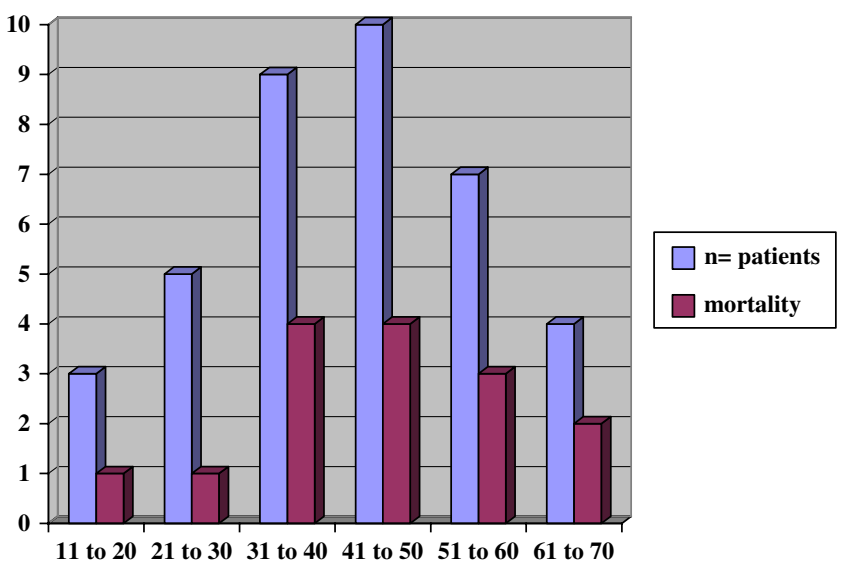

Figure 1. Age distribution of patients with Hantavirus infection supported with ECMO. 
time that preparations were being made for cannulation for ECMO. In some patients we did not wait for a positive serology test, which takes 8 to 12 hours.

\section{Cannulation and Decannulation Technique}

An attempt was made to insert the cannulas percutaneously into the femoral vessels whenever possible. In patients who were in hemodynamically stable condition and not yet intubated, a 16gauge/7-inch long catheter (Arrow arterial/venous catheterization set; Arrow International, Inc, Reading, Pa) was inserted percutaneously into the common femoral artery and another into the common femoral vein, using local anesthesia and the Seldinger technique. Once the decision was made to cannulate for ECMO, the patient received an initial dose of heparin, 200 units $/ \mathrm{kg}$. Subsequently, a 0.038-inch/39-inch long guidewire, included with the BioMedicus percutaneous arterial femoral kit (Medtronic Inc, Minneapolis, Minn), was introduced into the previously placed catheter in the femoral artery. A Bio-Medicus femoral arterial cannula, ranging in size from $15 \mathrm{~F}$ to $21 \mathrm{~F} / 18-\mathrm{cm}$ long, was inserted over the guidewire and then connected to the arterial tubing from the ECMO machine. Likewise, a 0.038 -inch/71-inch long guidewire, provided with the Bio-Medicus percutaneous femoral kit, was introduced into the previously placed catheter in the femoral vein. A Bio-Medicus femoral venous cannula, ranging in size from $22 \mathrm{~F}$ to $29 \mathrm{~F} / 76-\mathrm{cm}$ long, was placed over the guidewire and advanced to the right atrium. It was then connected to the venous tubing of the ECMO machine. Eight patients with pulseless electrical activity and/or ventricular fibrillation were cannulated by the open technique during external cardiac compressions.

To prevent ischemia of the lower extremity, we used a size $8 \mathrm{~F}$ or $10 \mathrm{~F} / 10$-cm long Bio-Medicus pediatric arterial cannula with obturator for distal perfusion of the superficial femoral artery in 20 of the 23 patients cannulated with the open approach. In addition, a distal venous cannula, size $14 \mathrm{~F}$ to $20 \mathrm{~F}$, was inserted distally into the femoral vein in 5 patients to relieve venous congestion.

Five $(13 \%)$ patients had poor venous drainage because the venous cannula could not be advanced into the inferior vena cava by the femoral approach. In these patients, a Thin-Flex single-stage venous cannula (Edwards Lifesciences LLC, Irvine, Calif), ranging in size from $30 \mathrm{~F}$ to $36 \mathrm{~F}$, was advanced without difficulty into the right atrium through the right internal jugular vein, which was exposed via an oblique neck incision anterior to the sternocleidomastoid muscle.

Patients were connected to the ECMO machine (Cobe Cardiovascular, Arvada, Colo) with a Wolf custom-pack nonheparinized 3/8-inch tubing (Medtronic Inc, Minneapolis, Minn), with an Avecor membrane oxygenator (Medtronic) and a Bio-Therm heat exchanger (Medtronic). The initial ECMO pump settings included a flow of 4.0 to $5.0 \mathrm{~L} / \mathrm{min}$ delivered by a roller pump system and an $\mathrm{FIO}_{2}$ of 1.0. Anticoagulation was maintained with heparin, and the activated clotting time (ACT) was monitored hourly, with values ranging from 180 to 220 seconds. After a few days of ECMO, the inotropic agents and $\mathrm{FIO}_{2}$ were weaned, as the hemodynamic status and pulmonary function improved gradually. Subsequently, the patients were weaned from ECMO, and when the pump flow was less than $1 \mathrm{~L} / \mathrm{min}$ and 1-hour trials off ECMO were tolerated, ECMO support was discontinued. The ACT was maintained greater than 200 seconds during the trials off ECMO to prevent clotting of the cannulas. Clotting of the femoral artery or vein was not an issue, because the patients were kept fully anticoagulated until the cannulas were removed. The cannulas were removed by an open approach. An additional 5000 units of heparin were given intravenously if the ACT was less than 200 seconds. After removal of the arterial cannula, a Fogarty embolectomy catheter was passed distally to retrieve any possible clots. The common femoral artery was repaired primarily in 14 patients by continuous $6-0$ polypropylene suture or by a patch of autologous saphenous vein in 11 patients. Likewise, the common femoral vein was repaired primarily with continuous $6-0$ polypropylene suture or with a patch of autologous saphenous vein in 2 patients. Normal distal pulses were present after decannulation in all patients who survived ECMO.

\section{Statistical Analysis}

The Fisher exact test was used to compare several categorical variables associated with increased mortality during ECMO support (Table 2), and the unpaired Student $t$ test was used for comparison of continuous variables (Table 3 ).

\section{Results}

Duration of ECMO support averaged 132 hours (range: 67276 hours in survivors and 5-262 hours in nonsurvivors). After ECMO was discontinued, all patients remained intubated for an average of 7.1 days (range: 3-12 days), and 3 patients required a tracheostomy.

The hospital mortality was $39.5 \%$ (15 patients), of whom 13 patients died during ECMO support and 2 additional patients died of multiorgan system failure after ECMO was discontinued by family request. The overall survival was $60.5 \%$ (23/38 patients). There was no significant difference in mortality for patients supported with ECMO for more than 7 days $(5 / 11$ died, mortality $=45.4 \%)$ when compared with patients supported with ECMO for less than 7 days (10/ 27 died, mortality $=37 \%)(P=.753)$. Six $(75 \%)$ of the 8 patients who had cardiac arrest before cannulation died of hypoxic-ischemic encephalopathy, 3 of whom also had multiorgan system failure. Other causes of death included multiorgan system failure in another 5 patients and sepsis and renal failure in 4 patients. Our results indicate that pre-ECMO cardiac arrest, severe lactic acidosis, and severe hypotension

\section{TABLE 2. Categorical variables associated with increased mortality during ECMO support, compared by the Fisher exact test}

\begin{tabular}{lclr}
\hline \multicolumn{1}{c}{ Variable } & \multicolumn{1}{c}{ Survivors } & Nonsurvivors & $\boldsymbol{P}$ value \\
\hline Female gender & $12 / 21(57.1 \%)$ & $9 / 21(42.9 \%)$ & .789 \\
Male gender & $11 / 17(64.7 \%)$ & $6 / 17(35.3 \%)$ & .744 \\
Intubated before & $16 / 23(69.6 \%)$ & $9 / 15(60 \%)$ & .728 \\
$\quad$ transfer & & & \\
Pre-ECMO CPR & $2 / 23(8.7 \%)$ & $6 / 15(40 \%)$ & .039 \\
1994-1999 & $8 / 16(50 \%)$ & $8 / 16(50 \%)$ & .426 \\
2000-2006 & $7 / 22(68.2 \%)$ & $7 / 22(31.8 \%)$ & .426 \\
\hline
\end{tabular}

$C P R$, Cardiopulmonary resuscitation; ECMO, extracorporeal membrane oxygenation. Boldface indicates statistically significance. 
TABLE 3. Continuous variables associated with increased mortality during ECMO support, compared by the unpaired Student $t$ test.

\begin{tabular}{lccc}
\hline \multicolumn{1}{c}{ Variable } & Survivors & Nonsurvivors & $\boldsymbol{P}$ value \\
\hline Age & $11-62(\mathrm{x}=40.1)$ & $15-69(\mathrm{x}=44.7)$ & .329 \\
Weight & $50-103 \mathrm{~kg}(\mathrm{x}=75 \mathrm{~kg})$ & $55-107 \mathrm{~kg}(\mathrm{x}=81.8 \mathrm{~kg})$ & .177 \\
Pre-ECMO platelet count & $13,000-63,000(\mathrm{x}=35,700)$ & $12,000-43,000(\mathrm{x}=25,500)$ & .073 \\
Pre-ECMO MAP & $36-80(\mathrm{x}=60.9 \mathrm{~mm} \mathrm{Hg})$ & $0-74(\mathrm{x}=40.8 \mathrm{~mm} \mathrm{Hg})$ & .038 \\
Pre-ECMO pH & $6.9-7.48(\mathrm{x}=7.30)$ & $2.05-7.49(\mathrm{x}=7.32)$ & .893 \\
Pre-ECMO serum lactate & $2.0-18.2(\mathrm{x}=4.7 \mathrm{mmol} / \mathrm{L})$ & $32.0(\mathrm{x}=11.4 \mathrm{mmol} / \mathrm{L})$ & .002 \\
Pre-ECMO PaO & $28-195(\mathrm{x}=81)$ & $5-262(\mathrm{x}=65)$ & $.327 .7)$ \\
Hours on ECMO & $67-276(\mathrm{x}=135.2)$ & .753 \\
\hline
\end{tabular}

ECMO, Extracorporeal membrane oxygenation; MAP, mean arterial pressure. Boldface indicates statistically significant.

were associated with significantly increased mortality $(P<$ .05) (Tables 2 and 3).

All patients who were successfully weaned and decannulated survived and recovered completely and were discharged from the hospital after a mean hospital stay of 20.8 days (range: 10-39 days). We observed that survival improved during the last few years. Before January 2000, 8 $(50 \%)$ of 16 patients died, whereas $7(31.8 \%)$ of 22 patients died since January 2000. However, there was no significant statistical difference between the two time periods $(P=$ .426). Sixteen of the 23 survivors had an uneventful course on ECMO and no complications.

No difference in survival was observed when percutaneous and open techniques of cannulation were compared. Six $(40 \%)$ of the 15 patients cannulated percutaneously and $9(39.1 \%)$ of the 23 patients who had open cannulation died. Complications from percutaneous cannulation occurred in $4(26.6 \%)$ of 15 patients. Two (13.3\%) of 15 patients had a large retroperitoneal hematoma develop, and $2(13.3 \%)$ of 15 patients had lower extremity ischemia develop. Adequate leg perfusion was re-established with thrombectomy-embolectomy and after insertion of an additional cannula in the superficial femoral artery.

Complications from open femoral cannulation occurred in $8(34.8 \%)$ of 23 patients: severe bleeding occurred in 7 $(30.4 \%)$ of 23 patients, and $1(4.3 \%)$ patient required a leg amputation because of lower extremity ischemia despite insertion of a distal perfusion cannula. Six (50\%) of the 12 patients with cannulation complications did not survive: 3 $(100 \%)$ of 3 patients with leg ischemia and $3(42.8 \%)$ of 7 patients with severe bleeding died. In contrast, 9 (34.6\%) of the 26 patients without cannulation complications did not survive. However, the difference in mortality between the patients who had serious cannulation complications and those who did not was not statistically significant $(P=.80)$.

Severe bleeding necessitating re-exploration occurred in 7 (30.4\%) of 23 patients after open femoral cannulation, and the median number of transfused units of red blood cells and platelets was 21.4 (range: 6-65 units) and 18 (range: $2-83$ ), respectively. Bleeding originated around the venous cannula in 2 patients, at the arterial cannulation site in 2 patients, was due to partial dislodgment of the superficial femoral artery cannula in 2 patients, and was caused by needle holes in another patient. Three $(42.8 \%)$ of the 7 patients with severe bleeding from the cannulation site died, but there was no statistically significant difference in mortality when compared with 9 (34.6\%) of 26 patients without cannulation complications who did not survive $(P=.80)$.

Other serious complications that we observed during ECMO support included mechanical problems in 3 patients who did not survive (tubing rupture in 2 and pump malfunction in 1) and patient complications (sepsis and renal failure in 6 patients, 2 of whom survived, and multiorgan system failure in 8 patients, who did not survive).

\section{Discussion}

HCPS resulting from SNV infection is characterized by 4 clinical phases: prodrome, pulmonary edema and shock, diuretic phase, and convalescence. ${ }^{6}$ Clinical presentation during the prodromal phase is characterized by high fever, headache, and myalgias for an average of 3 to 6 days, followed by dyspnea, which is frequently a sign of impending respiratory failure. The onset of respiratory distress and shock is abrupt. Risk of death is highest during the initial 24 hours of the pulmonary edema and shock phase of the illness, which also lasts from 3 to 6 days.

Despite early diagnosis of HCPS, improvement in critical care management, including mechanical ventilation, and hemodynamic support with vasoactive drugs, mortality rates range from $43 \%$ to $76 \% .^{5,6}$ Some patients, however, have a worse prognosis than others and are unlikely to survive despite aggressive medical management. Crowley and colleagues ${ }^{7}$ identified several criteria for nonsurvival, which include refractory shock, severe hypoxia $\left(\mathrm{PaO}_{2} / \mathrm{FIO}_{2}\right.$ ratio $<$ 60 ), and external cardiac compressions. In our present study, only those patients with a typical clinical presentation consistent with HCPS, a cardiac index that rapidly dropped to less than $2.0 \mathrm{~L} \cdot \min ^{-1} \cdot \mathrm{m}^{-2}$ despite maximum inotropic support, and at least one of the predictive criteria for nonsurvival mentioned above were considered for ECMO support. Early 
diagnosis of Hantavirus infection is strongly suspected by characteristic hematologic findings, including thrombocytopenia, hemoconcentration, leukocytosis, and the presence of at least $10 \%$ immunoblasts in the peripheral blood smear. ${ }^{8}$ The diagnosis of HCPS is confirmed by the presence of SNV antibodies. However, a major limitation of the serologic test is that it usually takes 8 to 12 hours, whereas a recombinant immunoblot assay allows the diagnosis within 4 hours. ${ }^{6}$ In contrast to patients with other forms of septic shock, patients with HCPS have a decreased cardiac index and elevated systemic vascular resistance. ${ }^{9}$ Although the usual chest radiographic findings include interstitial edema early in the disease course and lack of peripheral distribution of air space disease,${ }^{10}$ the hemodynamic parameters in HCPS are unique because the pulmonary artery wedge pressure is low, unlike other forms of cardiogenic shock, and these patients fail to improve with intravascular volume repletion, unlike other forms of hypovolemic shock. ${ }^{6}$

Treatment of HCPS during the pulmonary edema and shock phase poses a dilemma, because severe pulmonary capillary leak develops during volume administration. Inotropic support with dobutamine and mechanical ventilation are indicated during this phase to improve cardiac output and oxygenation. However, some patients fail to improve, and lactic acidosis and pulseless electrical activity develop rapidly.

Venoarterial cannulation for ECMO was used to treat our patients with HCPS because it provides support for both cardiac and respiratory failure. Venovenous ECMO is an alternative method of extracorporeal support for patients with severe respiratory failure who do not require cardiac support, but it is not applicable to treat patients with HCPS. A roller pump was used for all patients supported with ECMO, mainly because it provides a constant flow and is associated with a reduced risk of hemolysis. ${ }^{11}$

Vascular access can be accomplished percutaneously or by direct cutdown of the common femoral artery and vein. As an initial step before cannulation, we prefer to introduce percutaneously a guidewire and a sheath in the common femoral artery and another in the common femoral vein in patients who are in hemodynamically stable condition. This simple maneuver facilitates percutaneous insertion of the cannulas into the femoral vessels once the decision is made to cannulate for ECMO. However, cannulation of the femoral vessels was achieved percutaneously in only 15 (39.5\%) patients, and an open technique was required in the other $23(60.5 \%)$ patients. Cannulation during cardiac massage (8 patients) and difficulty inserting the cannula percutaneously into the femoral vein were the most common reasons requiring open cannulation. Axillary cannulation was not considered because it is more time consuming and may lead to more bleeding complications.

Poor venous drainage was usually corrected by repositioning the tip of the venous cannula in the right atrium.
Additional cannulation of the right internal jugular vein was required in $5(13.1 \%)$ patients because the venous cannula could not be advanced into the inferior vena cava by the femoral approach. Insertion of an additional venous cannula in the distal femoral vein, as described by Le Guyader and colleagues, ${ }^{12}$ was required in 5 patients to relieve venous leg congestion.

Ischemic injury of the lower extremity is a potential complication associated with prolonged ECMO support. In our experience, leg ischemia occurred in $3(7.9 \%)$ patients, of whom 2 were cannulated percutaneously. To prevent this serious complication, we used to insert a distal perfusion cannula in the superficial femoral artery in all patients cannulated with the open technique, as recommended by several authors. ${ }^{13,14}$ More recently, we have used a distal perfusion cannula in the posterior tibial artery inserted under direct vision in all patients. Huang and colleagues ${ }^{15}$ use a pressure criterion to select the patients at risk of distal leg ischemia and place a perfusion catheter if the distal mean pressure is below $50 \mathrm{~mm} \mathrm{Hg}$. An alternative method of femoral arterial cannulation using a single right-angle cannula, which maintains adequate bidirectional arterial perfusion, was described by Read and associates. ${ }^{16}$

Bleeding is the most common complication of ECMO, mainly because of systemic heparinization. ${ }^{17}$ Thrombocytopenia and platelet dysfunction are incremental risk factors for surgical bleeding in patients with Hantavirus infection. Severe hemorrhage may lead to profound hemodynamic decompensation during ECMO. Management of bleeding includes volume-for-volume replacement of blood loss, platelet transfusion to more than $125,000 / \mathrm{mm}^{3}$, and decreasing the ACT to 180 seconds. Epsilon aminocaproic acid, aprotinin, and tranexamic acid have recently been reported to minimize bleeding complications in patients supported by ECMO. ${ }^{17}$ Despite aggressive treatment of the underlying coagulopathy, surgical re-exploration of the cannulation site is usually necessary. According to the Registry Report 2004 of the Extracorporeal Life Support Organization, cannulation site bleeding occurred in $22 \%$ and $29 \%$ of adult patients supported by ECMO for respiratory failure and cardiac support, respectively. ${ }^{18}$ Magovern and Simpson ${ }^{19}$ reported that bleeding was noted in most patients with postcardiotomy cardiogenic shock supported by ECMO, $48 \%$ required reoperation for femoral vessel repair, and the average number of units of red blood cells and platelets transfused was 29 and 36 , respectively. In our series, severe bleeding necessitating re-exploration occurred in $30 \%$ of patients after open femoral cannulation, a large retroperitoneal hematoma developed after percutaneous cannulation in $13 \%$ of patients, and the average number of units of red blood cells and platelets transfused was 21 and 18, respectively. Although the bleeding complications might have been easier to manage with a centrifugal pump and heparin-coated circuits, the tradeoff is that centrifugal pumps do not have servo-regulation, which 
may lead to irregular flow delivery, and are also associated with a significantly increased risk of hemolysis. ${ }^{11}$

The overall survival in our series was $60.5 \%(23 / 38$ patients). By comparison, survival of adults supported by ECMO for postcardiotomy cardiogenic shock was $36 \%$ in the Allegheny experience ${ }^{19}$; it was $40 \%$ for adult cardiac failure and $54 \%$ for adult respiratory failure as reported in the 2004 Extracorporeal Life Support Organization Registry. ${ }^{18}$

In conclusion, almost two thirds of the patients with severe HCPS who were supported with ECMO survived and recovered completely. The complications associated with both types of femoral cannulation may be attributed to the fact that all patients were in shock or in full cardiac arrest, and the procedure had to be done expeditiously. Earlier institution of ECMO and refinement of cannulation techniques may decrease the complication rates and improve the overall survival.

We are very grateful to Cameron Crandall, $\mathrm{MD}, \mathrm{PhD}$ (Research Director, Department of Emergency Medicine, University of New Mexico Health Sciences Center), for the statistical analysis; to Patrick Kelly, John Clement, and Will Cabaniss (Perfusion Services at University of New Mexico Health Sciences Center); to Denise Coleman, MD, and Robert Katz, MD (Department of Pediatrics, University of New Mexico Health Sciences Center); and to Gary Iwamoto, MD, Michel Boivin, and Michele Harkins, MD (Division of Critical Care Medicine, Department of Internal Medicine, University of New Mexico Health Sciences Center).

\section{References}

1. Nichol ST, Spiropoulou CF, Morzunov S, Rollin PE, Ksiazek TG, Feldmann $\mathrm{H}$, et al. Genetic identification of a hantavirus associated with an outbreak of acute respiratory illness. Science. 1993;262:914-7.

2. Ye C, Prescott J, Nofchissey R, Goade D, Hjelle B. Neutralizing antibodies and Sin Nombre virus RNA after recovery from hantavirus cardiopulmonary syndrome. Emerg Infect Dis. 2004;10:478-82.

3. Childs JE, Ksiazek TG, Spiropoulou CF, Krebs JW, Morzunov S, Maupin GO, et al. Serologic and genetic identification of Peromyscus maniculatus as the primary rodent reservoir for a new hantavirus in the southwestern United States. J Infect Dis. 1994;169:1271-80.
4. Ferrés M, Vial P. Hantavirus infection in children. Curr Opin Pediatr. 2004; $16: 70-5$

5. Young JC, Hansen GR, Graves TK, Deasy MP, Humphreys JG, Fritz CL, et al. The incubation period of Hantavirus pulmonary syndrome. Am J Trop Med Hyg. 2000;62:714-7.

6. Simpson SQ. Hantavirus pulmonary syndrome. Heart Lung. 1998;27: 51-7.

7. Crowley MR, Katz RW, Kessler R, Simpson SQ, Levy H, Hallin G, et al. Successful treatment of adults with severe Hantavirus pulmonary syndrome with extracorporeal membrane oxygenation. Crit Care Med. 1998;26:409-14.

8. Koster F, Foucar K, Hjelle B, Scott A, Chong YY, Larson R, et al. Rapid presumptive diagnosis of Hantavirus cardiopulmonary syndrome by peripheral blood smear review. Am J Clin Pathol. 2001;116:665-72.

9. Hallin GW, Simpson SQ, Crowell RE, James DS, Koster FT, Mertz GJ, et al. Cardiopulmonary manifestations of hantavirus pulmonary syndrome. Crit Care Med. 1996;24:252-8.

10. Ketai LH, Williamson MR, Telepak RJ, Levy H, Koster FT, Nolte KB, et al. Hantavirus pulmonary syndrome: radiographic findings in 16 patients. Radiology. 1994;191:665-8.

11. Hansell DR. ECLS equipment and devices. In: Van Meurs K, Lally KP, Peek G, Zwischenberger JB, editors. Extracorporeal cardiopulmonary support in critical care. 3rd ed. Ann Arbor: Extracorporeal Life Support Organization; 2005. p. 107-19.

12. Le Guyader A, Lacroix P, Ferrat P, Laskar M. Venous leg congestion treated with distal venous drainage during peripheral extracorporeal membrane oxygenation. Artif Organs. 2006;30:633-5.

13. Greason KL, Hemp JR, Maxwell JM, Fetter JE, Moreno-Cabral RJ. Prevention of distal limb ischemia during cardiopulmonary support via femoral cannulation. Ann Thorac Surg. 1995;60:209-10.

14. Ceviz M, Ozyazicioglu A, Koçak H. The prevention of leg ischemia in femoral cannulation. Ann Thorac Surg. 1999;67:1217.

15. Huang SC, Yu HY, Ko WJ, Chen YS. Pressure criterion for placement of distal perfusion catheter to prevent limb ischemia during adult extracorporeal life support. J Thorac Cardiovasc Surg. 2004;128:776-7.

16. Read R, St Cyr J, Tornabene S, Whitman G. Improved cannulation method for extracorporeal membrane oxygenation. Ann Thorac Surg. 1990;50:670-1.

17. DeBerry BB, Lynch J, Chung DH, Zwischenberger JB. Emergencies during ECLS and their management. In: Van Meurs K, Lally KP, Peek G, Zwischenberger JB, editors. Extracorporeal cardiopulmonary support in critical care. 3rd ed. Ann Arbor: Extracorporeal Life Support Organization; 2005. p. 133-56.

18. Conrad SA, Rycus PT, Dalton HJ. Extracorporeal Life Support Registry Report 2004. ASAIO J. 2005;51:4-10.

19. Magovern GJ, Simpson KA. Extracorporeal membrane oxygenation for adult cardiac support: the Allegheny experience. Ann Thorac Surg. 1999;68:655-61. 